\title{
The influence of rubber crumbs on the critical state behavior of waste mixtures
}

\author{
Buddhima Indraratna ${ }^{1, *}$, Yujie Qi ${ }^{1}$, Ana Heitor ${ }^{1}$, and Jayan S. Vinod ${ }^{1}$ \\ ${ }^{1}$ University of Wollongong,Centre for Geomechanics and Railway Engineering (CGRE) and ARC Training Centre for Advanced \\ Technologies in Rail Track Infrastructure (ITTC-Rail), NSW 2522, Australia
}

\begin{abstract}
The practical application of waste materials such as steel furnace slag (SFS) and coal wash (CW) is becoming more prevalent in many geotechnical projects. It was found that the inclusion of rubber crumbs (RCs) from recycled tyres into mixtures of SFS and CW not only solves the problem of large stockpiles of waste tyres, it also can provide an energy-absorbing medium that will reduce track degradation. In order to investigate the influence of $\mathrm{RC}$ on the geotechnical properties of the granular waste matrix (SFS+CW+RC), a series of monotonic consolidated drained triaxial tests were conducted on waste mixtures. The test results reveal that the inclusion of RC significantly affects the geotechnical properties of the waste mixtures, especially their critical state behaviour. Specifically, the waste matrix can achieve a critical state with a low RC content $(<20 \%)$, whereas those mixtures with higher RC contents $(20-40 \%)$ cannot attain a critical state within the ultimate strain capacity that can be applied to specimens using the traditional triaxial equipment. Therefore, for the waste matrix with higher RC contents extrapolation of the measured volumetric strains had to be adopted to obtain the appropriate critical state parameters. Moreover, the influence of energy absorbing property by adding RC on the critical state behaviour has also been captured through an empirical equation.
\end{abstract}

\section{Introduction}

The stockpiles of end-of-life tyres keep mounting worldwide. It was reported that around 51 million equivalent passage units of waste tyres were generated in 2013-14 period alone in Australia, which exceeds 400,000 tonnes, of which only about $5 \%$ was properly recycled [1]. Most scrap tyres are normally placed in landfills or stockpiled on otherwise usable land areas. Stockpiling of waste tyres is highly undesirable because of the risk of combustion under high heat, health risks (e.g. breeding of mosquitoes) and obvious environmental pollution. Therefore, from both environmental and economical perspectives, there is a pressing need to encourage innovative recycling solutions of scrap tyres.

Rubber crumbs (RC) from scrap tyres have been reported to have high permeability, low unit weight, and high damping properties [2-7]. The high damping characteristics of rubber materials have made them promising materials to be included in geo-infrastructure to increase energy absorbing capacity (increased ductility), and reduce vibration and noise in transport corridors. In the past, numerous laboratory tests have been conducted on the rubber-soil mixtures, and it has been found that around $40-60 \%$ of vibration could be reduced by mixing rubber crumbs with soil under seismic conditions [8]. Sol Sánchez et al. [9] found that the inclusion of rubber crumbs within a ballast assembly could effectively reduce particle degradation attributed to increased energy retention capacity through increased ductility. Senetakis et al. [4] stated that the damping property of sand-rubber mixtures could be enhanced by increasing the rubber content, while Indraratna et al. [5] demonstrated the role of a synthetic energy absorbing layer (SEAL) is in lieu of conventional capping material (subballast) by mixing 10\% RC with steel furnace slag (SFS) and coal wash (CW).

However, the investigation of the influence of $\mathrm{RC}$ content $\left(R_{b}, \%\right)$ on the critical state behaviour of the rubber-soil mixtures from a fundamental (mathematical) insight is limited. Mashiri et al. [3] found that sand-tyre chips could not achieve a critical state even after substantial axial strains $(>20 \%)$. While rubber crumbs behave differently to rubber chips/fibre, the critical state behaviour of soil-RC mixtures is also influenced by other factors such as the shapes and sizes of the blended rubber elements [10-11]. Youwai \& Bergado [12] indicated when $R_{b}>30 \%$, the sand-RC mixtures could barely obtain the critical state (constant volumetric strain) under laboratory testing, so the condition at the end of the test was often regarded as a quasi-critical state. In order to better understand the critical state behaviour of SFS $+\mathrm{CW}+\mathrm{RC}$ mixtures, in this paper, the influence of $R_{b}$ on the critical state behaviour of the waste mixtures will be elaborated in detail. The relationship between the energy absorbing capacity and the critical state parameters will be explored and quantified.

\footnotetext{
* Corresponding author: indra@uow.edu.au
} 


\section{Materials and test program}

\subsection{Materials}

Coal wash, CW $\left(G_{s}=2.11\right)$ and steel furnace slag, SFS $\left(G_{S}=3.43\right)$ are waste byproducts produced in local coal mining and steel industry in Wollongong area. $\mathrm{RC}\left(G_{s}=\right.$ 1.15) are granulated waste tyre products available commercially. Fig. 1 shows the particle size distribution (PSD) curves of these three waste mixtures as well as the target PSD of the SFS $+\mathrm{CW}+\mathrm{RC}$ mixtures. In order to attain the target PSD, three different sizes of RC were used (Fig. 1). Before blending the waste materials, they were sieved and separated according to different size ranges. When mixing, the waste material with the exact mass according to different size was weighed and mixed thoroughly until a uniform appearance was obtained.

In this study, a blending ratio of SFS:CW=7:3 (by weight) as optimized by Indraratna et al. [5] was used, because with this blending ratio the waste mixtures could have sufficient shear strength with minimum swelling potential. $R_{b}=0,10,20,30$, and $40 \%$ by weight were used to evaluate the influence of rubber crumbs on the critical state behaviour of the waste mixtures.

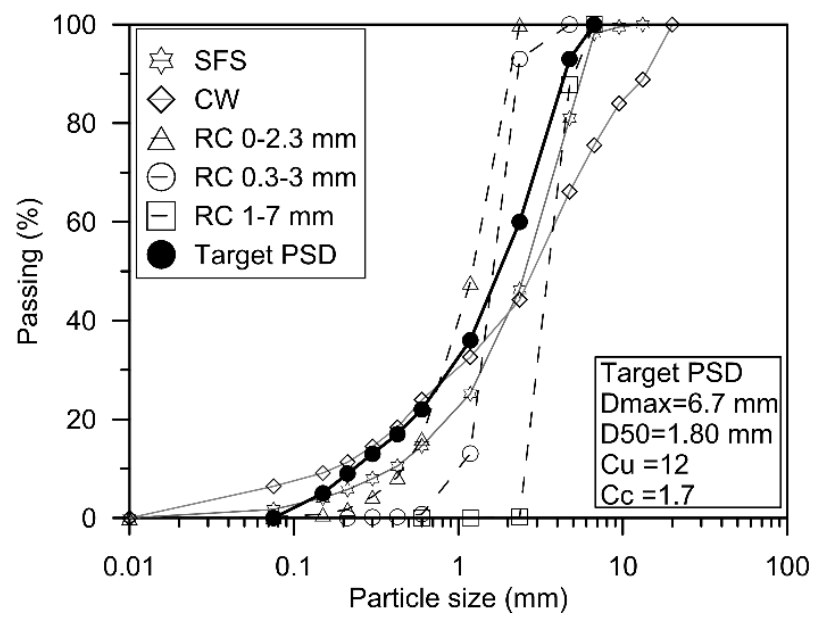

Fig. 1. PSD of waste materials and the target PSD for the mixtures (modified after Indraratna et al. [5]).

\subsection{Test program}

The A series of monotonic, consolidated-drained triaxial tests were carried out on the SFS+CW+RC mixtures. The test specimens were typically $50 \mathrm{~mm}$ (diameter) $\times 100$ $\mathrm{mm}$ (height). All the specimens were prepared at the optimum water content and compacted to around $95 \%$ of the maximum dry density.

The test procedure followed ASTM D7181 [13] with three stages, namely, saturation, consolidation, and shearing. Firstly, the test specimen was flooded using deaired water to get rid of the air inside the sample, and then the specimen was saturated by increasing the back pressure with a rate of $1 \mathrm{kPa} / \mathrm{min}$ until Skempton's B value exceeded 0.98. After this stage, the effective confining pressure $\left(\sigma^{\prime}{ }_{3}=10,40\right.$, or $\left.70 \mathrm{kPa}\right)$ was applied, and this stage was completed when the change of the specimen volume became less than $3 \mathrm{~mm}^{3}$ within 5 minutes. Note that the effective confining pressure level used here was to simulate the field conditions of a traditional capping layer in heavy haul railroads. Static shearing was applied after consolidation with a relatively small shearing rate of $0.2 \mathrm{~mm} / \mathrm{min}$ to ensure the specimen maintained fully drained conditions. The static shearing was completed when an axial strain $\left(\varepsilon_{1}\right)$ of $25 \%$ was achieved. Membrane correction was applied following ASTM D7181 [13].

\section{Test results}

\subsection{Stress and strain curve}

The stress ratio $\left(q / p^{\prime}\right.$; where $q$ is the deviator stress and $p^{\prime}$ is the mean effective stress) and strain plots of $\mathrm{SFS}+\mathrm{CW}+\mathrm{RC}$ mixtures having different $R_{b}$ values under different magnitudes of $\sigma_{3}^{\prime}$ are shown in Figs. 2-3. It can be observed that under the same effective confining pressure, i.e. $\sigma_{3}^{\prime}=70 \mathrm{kPa}$, with the addition of $\mathrm{RC}$, the peak stress ratio of the waste mixture decreases and the specimen becomes significantly more contractive (Fig. $2 \mathrm{a}, \mathrm{b}$ ); this can be attributed to the relatively low shear strength and high compressibility of rubber. Moreover, the axial strain according to the peak stress ratio also increases as $R_{b}$ increases, and the ductility of waste mixtures also increases accordingly. This is a definite advantage of adding $\mathrm{RC}$, because, high ductility means that the waste mixture would undergo a larger plastic deformation before failure. For a certain RC content (i.e. $R_{b}=40 \%$; Fig. $3 \mathrm{a}, \mathrm{b}$ ), when the effective confining pressure $\sigma_{3}^{\prime}$ increases, the peak stress ratio decreases, and the sample behaves more contractively.

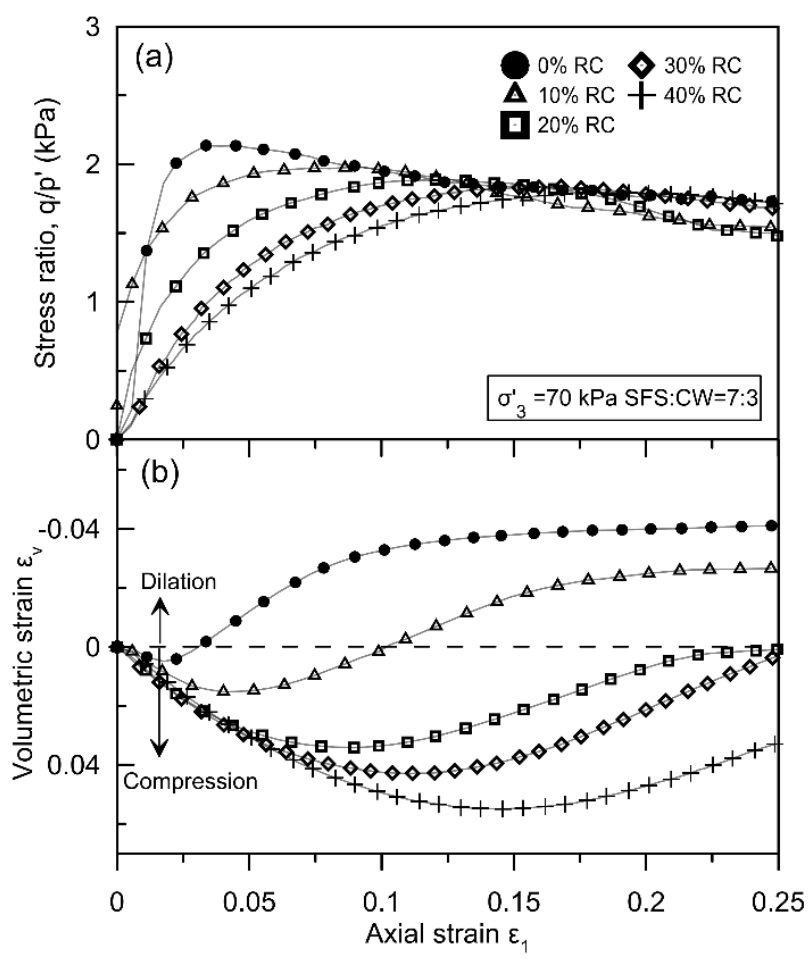

Fig. 2. Stress ratio-strain curves for $\mathrm{SFS}+\mathrm{CW}+\mathrm{RC}$ mixtures under $\sigma_{3}^{\prime}=70 \mathrm{kPa}$. 
It is noteworthy that the stress ratio and the volumetric strain $\varepsilon_{v}$ of SFS $+\mathrm{CW}+\mathrm{RC}$ mixture with $R_{b}=0.10 \%$ can easily stabilize before reaching $\varepsilon_{1}=25 \%$, while as $R_{b}$ increases the stabilization is harder to achieve (Fig. 2a, b). This is because more deformation occurs when a higher content of RC is included even at the end of the test. The same situation also occurs when increasing $\sigma_{3}^{\prime}$, i.e. the specimen having $R_{b}=40 \%$ tends to contract more towards the end of test (Fig. $3 \mathrm{~b}$ ), although the stress ratio and the volumetric strain are shown to change at a diminishing rate.

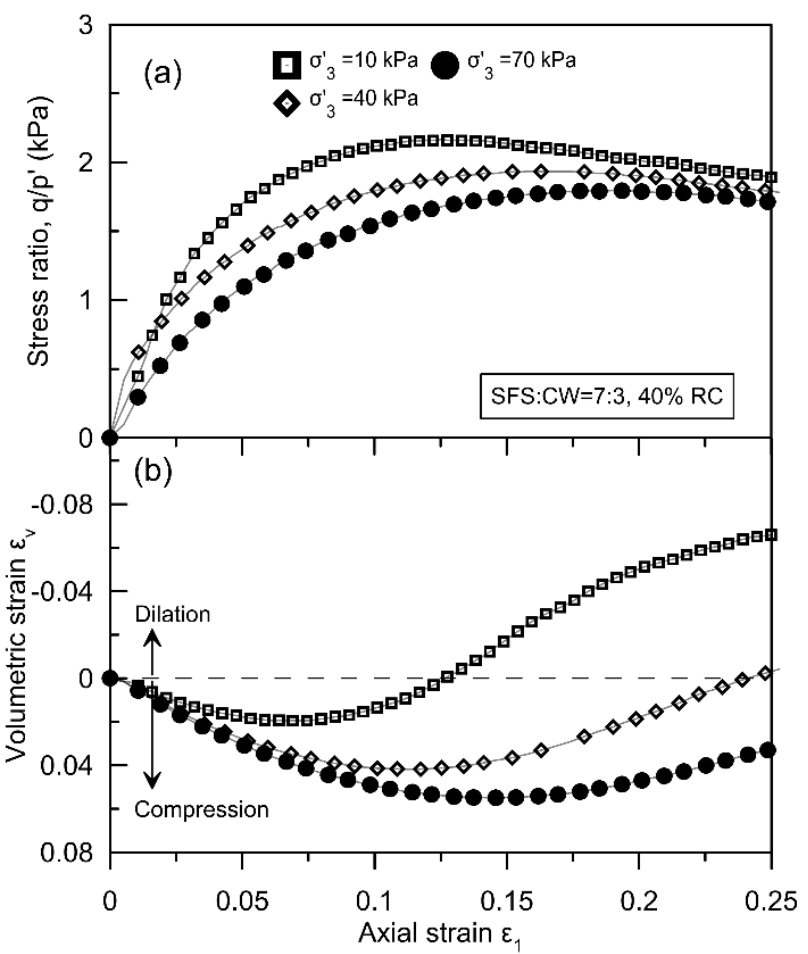

Fig. 3. Stress ratio-strain curves for $\mathrm{SFS}+\mathrm{CW}+\mathrm{RC}$ mixtures with $R_{b}=40 \%$.

\subsection{Critical state}

The critical state can be defined as when the soil reaches a condition at which both the deviator stress and the mean effective stress approach a constant value as the axial strain increases, and the corresponding soil dilatancy $\left(d \varepsilon_{v}^{p} / d \varepsilon_{q}^{p}\right)$ also reaches zero, i.e.

$$
\frac{d q}{d \varepsilon_{q}}=\frac{d p}{d \varepsilon_{q}}=\frac{d \varepsilon_{v}^{p}}{d \varepsilon_{q}^{p}}=0
$$

Where, $\varepsilon_{q}$ is the deviator strain, $\varepsilon_{v}^{p}$ is the plastic volumetric stain, and $\varepsilon_{q}^{p}$ is the plastic deviator strain.

Mashiri et al. [3] found that the sand-tyre chip mixtures could not reach a critical state as tyre chips would continually deform even at large axial strains. This is not surprising as tyre chips are relatively coarse aggregates and behave more like a composite material containing polymeric fibers or steel fibers [10-11]. In contrast, rubber crumbs are smaller granular materials and behave more like a uniform single material. When RC is mixed with other rigid materials (i.e. SFS and CW), the extent of RC-to-RC particle contacts will decrease, but further deformation of RC upon loading may be prevented by the surrounding rigid particles. Therefore, theoretically, the soil-RC mixtures should reach a critical state, although the axial strain of the triaxial tests would exceed $25 \%$, which is often the limit of most triaxial equipment.

In this study, when $R_{b}=0 \%$ and $10 \%$, the $\mathrm{SFS}+\mathrm{CW}+\mathrm{RC}$ mixtures could reach the critical state under the laboratory conditions, whereas when $R_{b} \geq$ $20 \%$, critical state could not be attained within the test limit of $\varepsilon_{1}=25 \%$. Therefore, extrapolation was applied to obtain the critical state for these waste mixtures for $R_{b} \geq 20 \%$ (Fig. 4).
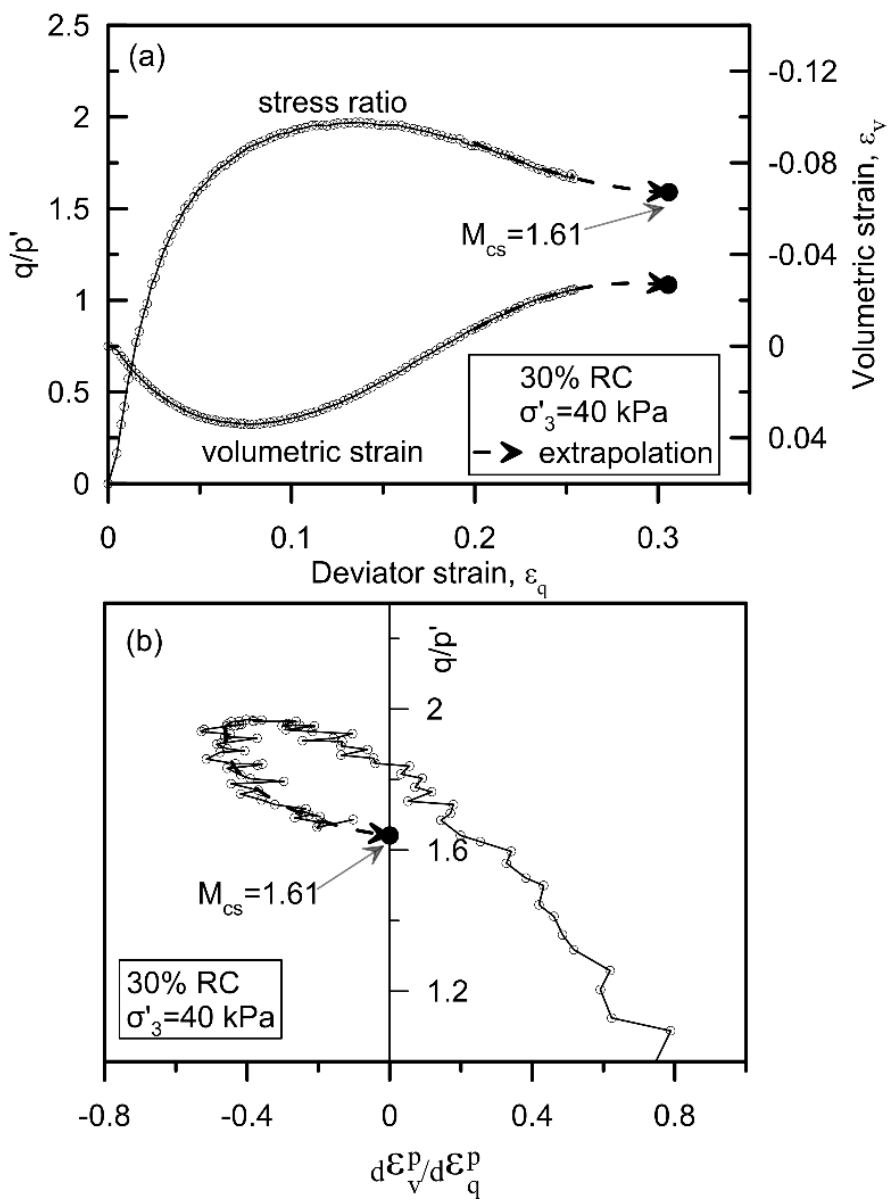

Fig. 4. Obtain critical state parameter through extrapolation.

This method of extrapolation to determine the critical state parameters of a fine granular material (tailings) was vividly described by Carrera et al. [14] and by Modoni et al. [15] for compacted gravel, and subsequently extended to coarse granular materials (ballast) by Indraratna et al. [16]. Fig. 4 shows an example for $\mathrm{SFS}+\mathrm{CW}+\mathrm{RC}$ mixtures having $30 \% \mathrm{RC}$. The critical stress ratio $\left(M_{c S}\right)$ here is obtained by extending the stress ratio-dilatancy plot to the intersection point with the zero dilatancy axes. The $q / p^{\prime}-\varepsilon_{q}$ and $\varepsilon_{v}-\varepsilon_{q}$ curves are also extrapolated to the most probable point for reaching zero increment of volumetric strains. In this way, the critical state parameters for the current waste mixtures with $R_{b}=$ $20 \%$ and $40 \%$ could be obtained. The critical stress ratio $\left(M_{c S}\right)$ and the corresponding void ratio $\left(e_{c S}\right)$ of $\mathrm{SFS}+\mathrm{CW}+\mathrm{RC}$ mixtures are given in Table 1 . 
Table 1. Critical parameters and the total work input of $\mathrm{SFS}+\mathrm{CW}+\mathrm{RC}$ mixtures.

\begin{tabular}{|c|c|c|c|c|c|c|}
\hline $\begin{array}{c}\text { Mixtures } \\
\text { SFS:CW= } \\
7: 3\end{array}$ & $\begin{array}{c}\boldsymbol{\sigma}^{\prime}{ }_{3} \\
(\mathrm{kPa} \\
)\end{array}$ & $M_{c s}$ & $e_{c s}$ & $\begin{array}{c}\boldsymbol{W}_{\text {total }} \\
(\mathrm{kPa})\end{array}$ & $\Gamma$ & $\lambda$ \\
\hline \multirow{3}{*}{$0 \% \mathrm{RC}$} & 10 & 1.94 & 0.40 & 2.18 & \multirow[t]{3}{*}{0.61} & \multirow{3}{*}{0.062} \\
\hline & 40 & 1.80 & 0.33 & 9.02 & & \\
\hline & 70 & 1.74 & 0.28 & 10.79 & & \\
\hline \multirow{3}{*}{$10 \% \mathrm{RC}$} & 10 & 2.01 & 0.34 & 4.84 & \multirow[t]{3}{*}{0.74} & \multirow[t]{3}{*}{0.101} \\
\hline & 40 & 1.61 & 0.29 & 14.80 & & \\
\hline & 70 & 1.55 & 0.24 & 31.18 & & \\
\hline \multirow{3}{*}{$20 \% \mathrm{RC}$} & 10 & 1.98 & 0.42 & 5.95 & \multirow[t]{3}{*}{0.89} & \multirow[t]{3}{*}{0.141} \\
\hline & 40 & 1.55 & 0.26 & 18.66 & & \\
\hline & 70 & 1.51 & 0.20 & 33.82 & & \\
\hline \multirow{3}{*}{$30 \% \mathrm{RC}$} & 10 & 2.0 & 0.40 & 5.46 & \multirow[t]{3}{*}{0.94} & \multirow[t]{3}{*}{0.159} \\
\hline & 40 & 1.61 & 0.24 & 21.93 & & \\
\hline & 70 & 1.48 & 0.16 & 38.58 & & \\
\hline \multirow{3}{*}{$40 \% \mathrm{RC}$} & 10 & 1.8 & 0.39 & 5.81 & \multirow[t]{3}{*}{1.01} & \multirow[t]{3}{*}{0.183} \\
\hline & 40 & 1.5 & 0.22 & 23.28 & & \\
\hline & 70 & 1.43 & 0.15 & 42.74 & & \\
\hline
\end{tabular}

The critical state parameters in Table 1 shows that $M_{C S}$ and $e_{c s}$ are not constant for each $\mathrm{SFS}+\mathrm{CW}+\mathrm{RC}$ mixture as they vary with $\sigma_{3}^{\prime}$ and $R_{b}$. Figs. 5a, b present the critical state lines of the waste mixtures in $q-p^{\prime}$ and $e-$ $p^{\prime}$ spaces, respectively. It can be seen that the critical state lines in $q-p^{\prime}$ space do not go through the origin as expected, and a small cohesion interception does occur, even for this granular material. This is also in line with Mashiri et al. [3] and Youwai \& Bergado [12], and the reason for this being that $M_{c s}$ is not a constant as assumed. Moreover, with the addition of $\mathrm{RC}$, the cohesion interception is expected to increase, which causes the critical state line to rotate clockwise, and the same trend applies to the critical state line in $e-p^{\prime}$ space (Fig. 5b). Note that a quasi-linear relationship can be established for the critical lines in $e-p^{\prime}$ space:

$$
e_{c s}=\Gamma-\lambda \ln p_{c s}^{\prime}
$$

where, $\Gamma$ is the void ratio when ${p^{\prime}}_{c s}=1 \mathrm{kPa}$, and $\lambda$ is the gradient of the critical state line in $e-p^{\prime}$ space.

It is interesting to observe that critical parameters $\Gamma$ and $\lambda$ show a linear relationship with the RC content (Fig. 5c) as follows:

$$
\begin{aligned}
& \Gamma^{*}=\Gamma_{1}+\Gamma_{2} R_{b} \\
& \lambda^{*}=\lambda_{1}+\lambda_{2} R_{b}
\end{aligned}
$$

where, $\Gamma^{*}$ and $\lambda^{*}$ are modified critical state parameters capturing $R_{b}$, while $\Gamma_{1}, \Gamma_{2}, \lambda_{1}$, and $\lambda_{2}$ are calibration parameters (Fig. 5c).

\subsection{The influence of energy absorbing capacity on critical state of the waste mixtures}

The inclusion of RC could significantly increase the energy absorbing capacity of the blended SFS $+\mathrm{CW}+\mathrm{RC}$ mixtures [5-6]. To capture the influence of the energy absorbing capacity on the critical state behavior of these waste mixtures, the total work input $\left(W_{\text {total }}\right)$ up to failure is examined. Note that the failure here is defined as the condition at which the specimen achieves its peak deviator stress, and this is the same as that of Zornberg et al. [18] for sand-tire shred mixtures.
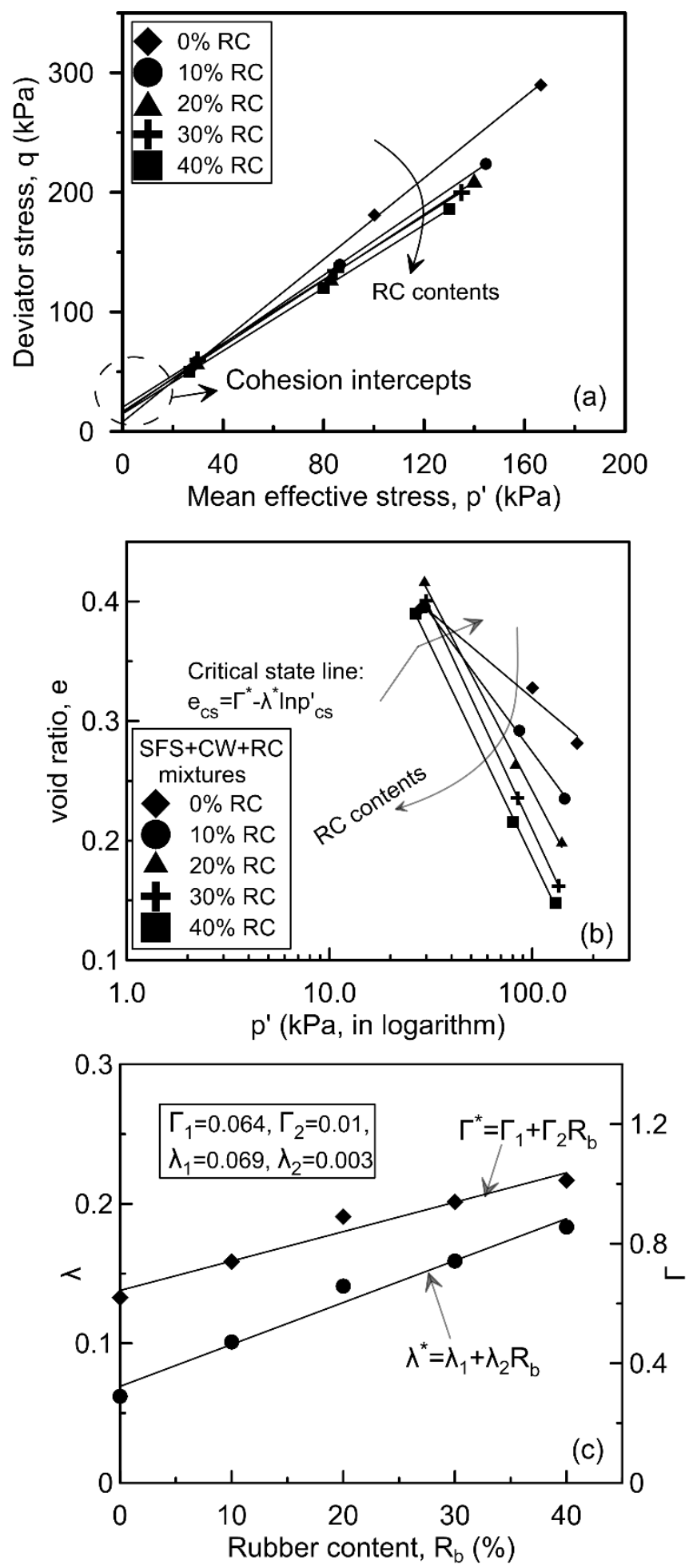

Fig. 5. (a) Critical state line in $q-p^{\prime}$ space; (b) Critical state line in $e-p^{\prime}$ space; (c) The influence of RC content on the critical state parameters (modified after Qi et al. [17]).

In general, the energy input to a unit volume of $\mathrm{SFS}+\mathrm{CW}+\mathrm{RC}$ specimen is consumed via two main phases, i.e. frictional work done through particles 
rearrangement, and the deformation of particles themselves. As the deformation of the rigid particles (i.e. SFS and CW) can be neglected, the energy consumed by particle deformation is mainly applicable for rubber crumbs. This also explains why rubber materials have a high energy absorbing capacity [2]. Two components can be distinctly considered in the total work, i.e. volumetric and deviatoric components, and by decoupling these two components, the increment of work applied onto the sample can be determined by [15]:

$$
d W_{\text {total }}=p^{\prime} d \varepsilon_{v}+q d \varepsilon_{q}
$$

The value of $W_{\text {total }}$ of the waste mixtures having different values of $R_{b}$ under different magnitudes of $\sigma_{3}^{\prime}$ is given in Table 1 . Note that, $W_{\text {total }}$ of the waste mixture increases with the increasing values of $\sigma_{3}^{\prime}$ and $R_{b}$. Thus $W_{\text {total }}$ is another parameter apart from $M_{c s}$ that would be influenced by $\sigma_{3}^{\prime}$ and $R_{b}$, indicating that $W_{\text {total }}$ may have a special relationship with $M_{C S}$.

Chavez \& Alonso [19] developed a model for rockfill in which the influence of suction and confining stress on $M_{c s}$ could be captured by introducing an additional factor, i.e. plastic work. In a similar way, the total work $W_{\text {total }}$ is then applied to represent the influence of $\sigma_{3}^{\prime}$ and $R_{b}$ on $M_{c s}$. By combining $W_{\text {total }}$ with $M_{c s}$ (Fig. 6), an empirical relationship can be developed as:

$$
M_{c s}^{*}=M_{0} *\left(\frac{W_{t o t a l}}{W_{0}}\right)^{\alpha}
$$

where, $M_{0}$ is the critical stress ratio when $W_{\text {total }}=$ $1 \mathrm{kPa}, \alpha$ is a regression coefficient, and $W_{0}=1 \mathrm{kPa}$ is used to keep the units of both sides of the equation the same. In this way, the energy absorbing property of RC is effectively translated through the total work input parameter, $W_{\text {total }}$.

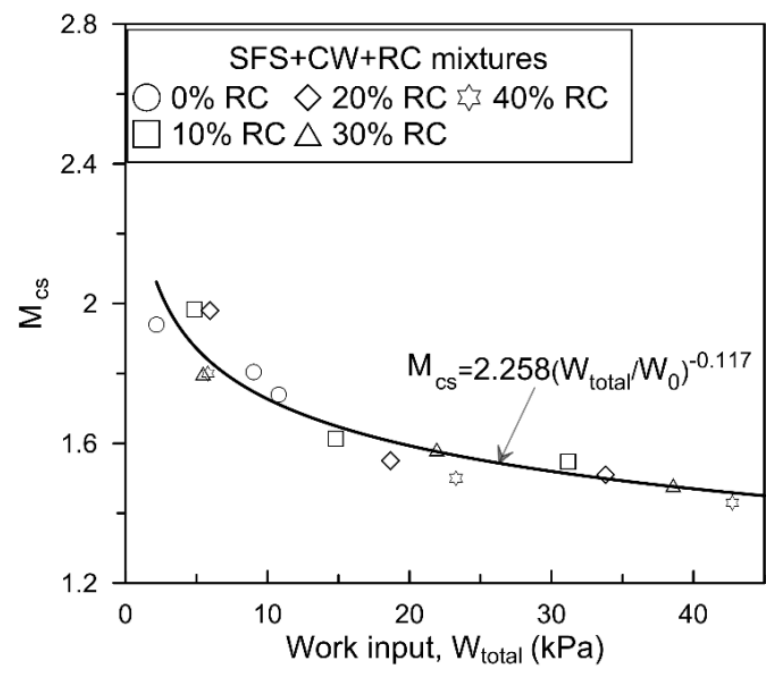

Fig. 6. The relationship between the critical state ratio and the total work input (modified after Qi et al. [17]).

\section{Conclusions}

In this paper, the critical state behaviour of the $\mathrm{SFS}+\mathrm{CW}+\mathrm{RC}$ mixtures was investigated through a series of monotonic, consolidated-drained triaxial tests. The laboratory results revealed that when increasing the RC content and the effective confining pressure, the peak deviator ratio decreased and the blended waste specimen behaved more contractively. Moreover, the inclusion of $\mathrm{RC}$ could significantly influence the critical state behavior of the waste mixtures. The critical state for SFS $+\mathrm{CW}+\mathrm{RC}$ mixtures with $R_{b}<20 \%$ could be easily achieved under the laboratory conditions, whereas for the waste mixtures with $R_{b} \geq 20 \%$ extrapolation was needed to obtain the suitable critical state parameters. The influence of energy absorbing capacity on the critical state behaviour of the waste mixtures was captured by introducing the total work input $W_{\text {total }}$, and an empirical relationship between $W_{\text {total }}$ and the critical state ratio $M_{c s}$ could be established.

The first author would like to acknowledge the financial assistance provided by the Australian Research Council (ARC) Discovery Project (ARC-DP) and ARC Industry Transformation Training Centre for Advanced Rail Track Technologies (ARCITTC-Rail). The assistance provided by industry (ASMS, South 32, and Tyre Crumbs Australia) in relation to the procurement of material used in this study is gratefully acknowledged. The laboratory assistance provided by Mr. Richard Berndt and the technical feedback from A/Professor Cholachat Rujikiatkamjorn are appreciated. Some figures in this paper have been modified, whereby the original data has been reproduced with kind permission from ASCE: Journal of Materials in Civil Engineering.

\section{References}

1. E. Mountjoy, D. Hasthanayake, T. Freeman. National Environmental Protection Council (2015)

2. Z.Y. Feng, G.K. Sutter. Geotechnical Testing Journal, 23(3), 338-344 (2000)

3. M. Mashiri, J.S. Vinod, M.N. Sheikh, H.H. Tsang. Soil and Foundations, 55(3), 517-528 (2015)

4. K. Senetakis, A. Anastasiadis, K. Pitilakis. J. Soil Dyn. Earthquake Eng., 33(1), 38-53 (2012)

5. B. Indraratna, Y. Qi, A. Heitor. J. Mater. Civ. Eng., 30(1), 04017251 (2018)

6. Y. Qi, B. Indraratna, A. Heitor, J.S. Vinod. Proceedings of the International Symposium on Geotechnics of Transportation Infrastructure (ISGTI 2018). New Delhi, India (2018)

7. Y. Qi, B. Indraratna, A. Heitor, J.S. Vinod. J. Geotech. Geoenviron. Eng., 144(2), 04017107 (2018)

8. H.H. Tsang, S.H. Lo, X. Xu, M.N. Sheikh. Earthquake Engineering and Structural Dynamics, 41(14), 2009-2024 (2012)

9. M. Sol-Sánchez, N.H. Thom, F. Moreno-Navarro, M.C. Rubio-Gamez, G.D. Airey. Construction and Building Materials, 75, 19-24 (2015) 
10. M. Mashiri, J.S. Vinod, M.N. Sheikh, J.A.H. Carraro. Environmental Geotechnics: 16.00016 (2017)

11. R. Fu, M.R. Coop, X.Q. Li. J. Geotech. Geoenviron. Eng. 143(9), 04017059 (2017)

12. S. Youwai, D.T. Bergado. Can. Geotech. J. 40(2), 254-264 (2003)

13. ASTM. American Society for Testing and Materials. D7181, West Conshohocken, PA (2011)

14. A. Carrera, M. Coop, R. Lancellotta. Géotechnique 61(11), 935-946 (2011)

15. G. Modoni, J. Koseki, L.Q. Anh Dan. Géotechnique 61(6), 473-485 (2011)

16. B. Indraratna, Q. Sun, S. Nimbalkar. Can. Geotech. J. 51, 1-14 (2015)

17. Y. Qi, B. Indraratna, J.S. Vinod. J. Mater. Civ. Eng. 30 (11), 04018276 (2018)

18. Zornberg, J.G., Viratjandr, C. \& Cabral, A.R. Can. Geotech. J. 41(2), 227-241 (2004)

19. C. Chavez, E.E. Alonso. Soil and Foundations 43(4), 215-227 (2003) 